\section{Asociación de polimorfismos de los genes CRF-BP, SLC6A4 y restricción alimentaria crónica: un estudio preliminar}

\author{
JORGE A. SANHUEZA ${ }^{1,2,3,4, a,}$, CHRISTIAN L. HERRERA ${ }^{2,3,4, b}$, \\ LUIS A. SALAZAR ${ }^{3,4, c}$, JAIME R. SILVA ${ }^{4,5, d}$
}

\section{CRF-BP and SLC6A4 gene polymorphisms among restrained eaters}

Background: Restrained eaters (RE) are a group of individuals who constantly restrict their eating. However, they usually alternate restriction with periods of overeating. Aim: To evaluate the possible association of CRF-BP and SLC6A4 gene polymorphisms with chronic alimentary restriction. Material and Methods: The Spanish version of the Revised Restraint Scale was applied to 132 women aged 18 to 25 years. They were divided in a group classified as restrained eaters (RE) and a group of unrestrained eaters. The 5-HTTLPR and CRF-BPs11 polymorphisms of the SLC6A4 and CRF-BP genes were evaluated by polymerase chain reaction (PCR) and PCR-restriction fragment length polymorphism (RFLP), respectively. Results: There was a significant association between the s/s homozygous genotype for the 5-HTTLPR polymorphism of SLC6A4 gene and RE condition ( $p=0.033$ ). However, this association was not observed for the CRF-BPs11 polymorphism. Conclusions: The presence of $s / \mathrm{s}$ genotype is associated with the RE condition, being the presence of a s allele, a risk factor for this condition.

(Rev Med Chile 2011; 139: 1261-1268).

Key words: Eating disorders; GABA plasma membrane transport proteins; Polymorphism, genetic.

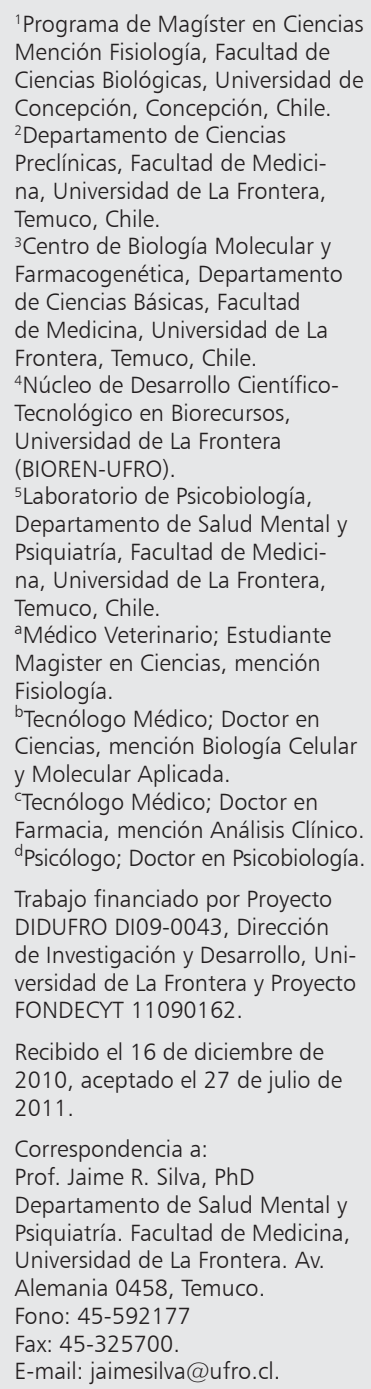

L os individuos dietantes crónicos (DC) son un grupo que si bien no conforman parte de una entidad patológica reconocida ${ }^{1,2}$, representan un grupo de riesgo a desarrollar patologías como la obesidad y los trastornos de la conducta alimentaria ${ }^{2-4}$. Los DC se caracterizan por presentar un temor exacerbado a subir de peso y continuamente restringen su alimentación sometiéndose a dietas ${ }^{2}$. Sin embargo, bajo ciertas condiciones ligadas a los estados de ansiedad, sintomatología depresiva y situaciones estresantes, buscan compensar estas situaciones mediante la sobrealimentación $\mathrm{n}^{2,3,5,6}$. Por ello, la restricción alimenticia y sus potenciales consecuencias (especialmente en condiciones de estrés crónico), pueden conformar una sucesión de estadios hacia el desarrollo de la obesidad ${ }^{2}$. Al parecer, los fenómenos psiconeuroendocrinológicos que se originan a partir del estrés crónico, y que se encuentran acentuados especialmente en personas crónicamente restringidas en su alimentación, favorecen el desarrollo progresivo de aumento de peso (obesidad central) y la cascada de eventos derivados ${ }^{2}$.

El modelo desarrollado por Silva y colaboradores $^{4}$ sugiere que los DC poseen un estilo afectivo 
vulnerable, el que implicaría una sensibilidad al estrés, un bajo umbral de activación de la ansiedad y una dificultad en regular las emociones negativas ${ }^{1,4,6,7}$. Esta propuesta puede ser descrita como un modelo de tres niveles. En un primer nivel, existirían diferentes configuraciones en la preponderancia de los sistemas motivacionales. A partir de sistemas motivacionales de activación asimétrica emergerían estilos afectivos de mayor o menor vulnerabilidad de desarrollo de psicopatología. En un segundo nivel, las diferencias en la vulnerabilidad afectiva se expresarían superficialmente, bajo condiciones de estrés y mediados por la factores socioculturales/familiares, en mecanismos de regulación afectiva basados en oscilaciones recurrentes de restricción/sobrealimentación. En un último nivel, la exacerbación de la restricción y/o sobreingesta, daría origen a trastornos de la conducta alimentaria y/u obesidad (Figura 1).

La hipótesis de la sensibilidad al estrés en $\mathrm{DC}^{1,4,5}$ busca develar evidencia en los distintos niveles de análisis del problema de la restricción alimentaria crónica. Actualmente, nuestro equipo de trabajo ha mostrado evidencia conductual al respecto $^{7}$, pero se requiere evidencia adicional en parámetros psicobiológicos. En este contexto, uno de los elementos biológicos clásicamente asociado a disfunciones de la respuesta al estrés, es el eje hipotálamo-hipófisis-adrenal (HHA) y los factores que regulan su funcionamiento. Dos de los principales reguladores del eje HHA son el sistema serotoninérgico y la proteína ligante del factor liberador de corticotrofinas (corticotropinreleasing factor binding protein; CRF-BP). Por ello, tal como se expondrá a continuación existe la posibilidad de explorar el rol de dichos factores en la condición de restricción alimentaria crónica.

El sistema serotoninérgico es un modulador de la conducta emocional en general y está ligado con rasgos como la ansiedad, depresión, conducta alimentaria e incluso la actividad reproductiva ${ }^{8}$. La modulación de la serotonina ocurre a nivel multisináptico regulando la actividad de otros transmisores, facilitando o regulando las sinap-

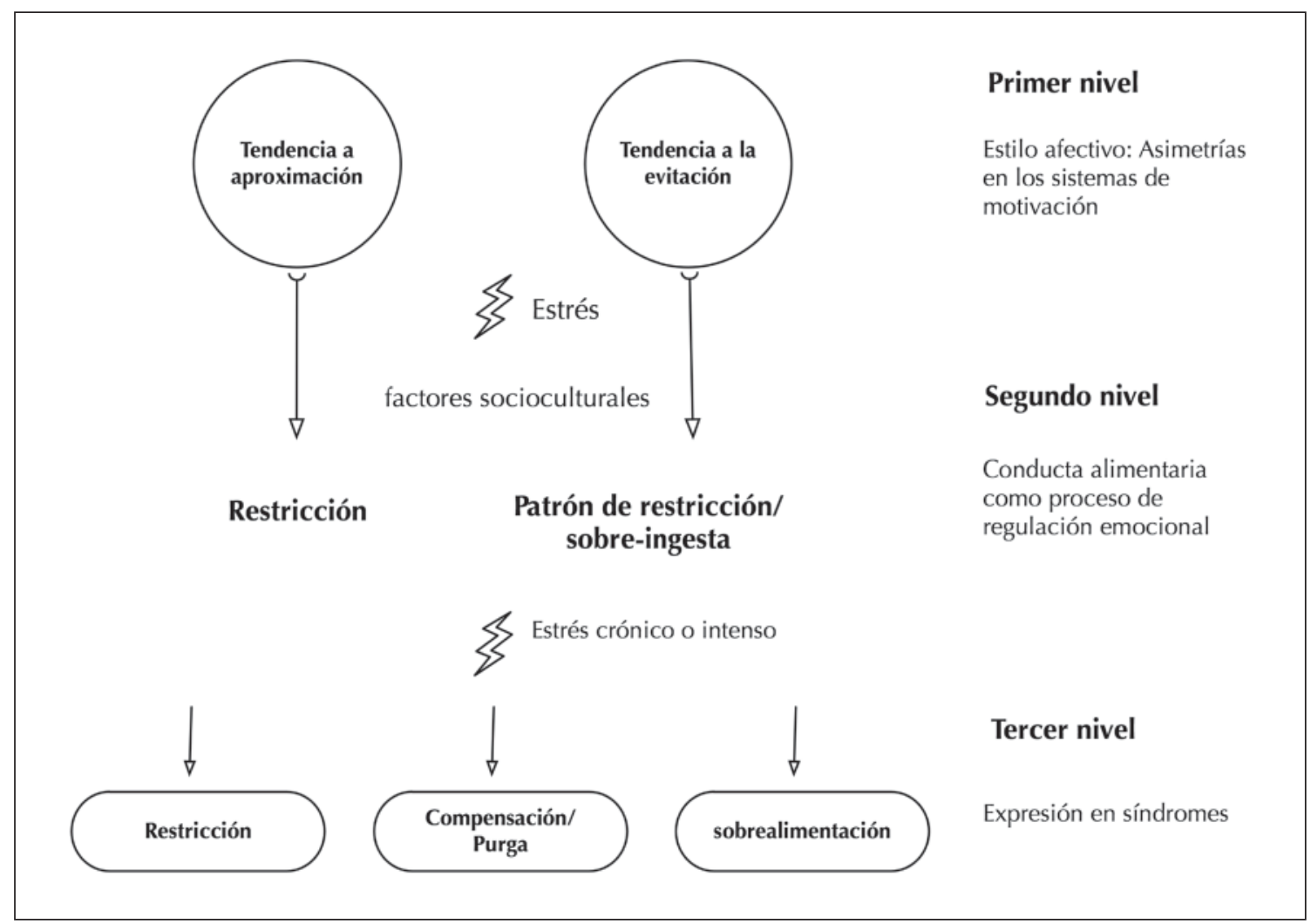

Figura 1. Modelo del estilo afectivo y conductas alimentarias problemáticas. 
Polimorfismos de CRF-BP y SLC6A4 y restricción alimentaria - J. A. Sanhueza et al

sis. Particularmente, el sistema serotoninérgico jugaría un rol clave en la regulación del eje HHA, regulando su función al menos en dos niveles; por un lado, activando a neuronas que liberan CRF; y por otro, regulando la actividad del CRF y cortisol a nivel sináptico. En este marco, el recaptador de serotonina (SERT) es quien regula las concentraciones de serotonina (5-HT) a nivel sináptico ${ }^{8-12}$.

Más de doce rasgos diferentes del comportamiento humano y otras patologías sistémicas han sido asociados a las variaciones del gen del SERT $(\mathrm{SLC6A4})^{13,14}$. La expresión reducida del gen y la función derivada de una variación importante en la región de control transcripcional de este (the serotonin.transporter-gene-linked polymorphic region; 5-HTTLPR), están ligadas a múltiples condiciones psicopatológicas, incluyendo las alimentarias ${ }^{8,14,15}$.

El gen SLC6A4 de humanos se encuentra en el cromosoma $17 \mathrm{q} 11.2$ y está compuesto por 14 exones que se extienden $40 \mathrm{~kb}^{16}$. La secuencia transcripcional permite la formación de una proteína de 630 aminoácidos con 12 dominios transmembrana. El polimorfismo de 5-HTTLPR corresponde a una variante genética en la cual ocurre una inserción-deleción de un fragmento de 44 pares de bases (bp) en el gen SLC6A4, donde la variante más corta (deleción) (short/short o $s / s$ ) resulta en menor actividad transcripcional y mayor vulnerabilidad a trastornos afectivos ${ }^{16-18}$. La variante génica se representa por un modelo bialélico ${ }^{16}$ aunque recientemente se ha documentado la presencia del un modelo trialélico ${ }^{19}$. Para el desarrollo de este estudio se utilizó la variante génica de 5-HTTLPR mediante el modelo bialélico.

Otro componente esencial de la fisiología de respuesta al estrés es el factor liberador de corticotrofinas (CRF) y su interacción con los receptores CRF1 y CRF2, los cuales regulan y modulan la actividad del eje HHA. La liberación de CRF desde el núcleo periventricular del hipotálamo, induce la liberación de adrecoticotrofina (ACTH) desde la adenohipófisis y la consecuente actividad de esta sobre la glándula suprarrenal para indicar la liberación de cortisol $^{20}$. Evidencia científica muestra que la regulación central de la liberación de CRF está mediada por la proteína ligante de CRF (CRF-BP), una proteína que liga al CRF y por ende su propiedad de inducir la liberación de ACTH, produciendo un feed-back adicional a la modulación del eje $\mathrm{HHA}^{21,22}$. Estudios muestran un incremento en la expresión de CRF-BP en la amígdala, hipófisis anterior y circulación portal luego de un incremento de la liberación de $\mathrm{CRF}^{22}$.

La CRF-BP es un polipéptido de 322 aminoácidos altamente conservado entre las especies. El gen de CRF-BP de $18 \mathrm{~kb}$, consiste en 7 exones y 6 intrones, y fue mapeado en el brazo largo del cromosoma $5^{23,24}$. Estudios muestran que la expresión de CRF-BP esta aumentada en la hipófisis y amígdala de ratas expuestas a estrés agudo, controlando la secreción aumentada de CRF. Además, en ratones knockout para CRF-BP se demostró un comportamiento ansioso y un incremento de la concentración de CRF y por ende los niveles de ACTH y cortisol ${ }^{22}$. Estudios preliminares sugieren que polimorfismos de las regiones intrónicas del gen que codifica CRF-BP (CRF-BPs2, CRF-BPs11 y CRF-BPs12), muestran asociación con el estado de depresión mayor ${ }^{25}$. Además, ratas con deficiencia de CRF-BP muestran una conducta anoréxica y ansiedad exacerbada ${ }^{26}$. Estas investigaciones podrían relacionar polimorfismos genéticos del gen que codifica CRF-BP, con alteraciones en la regulación del eje HHA, y con alteraciones conductuales asociadas a la alimentación.

En el contexto conceptual antes descrito, proponemos desarrollar un programa de investigación que evalúe el rol general y la asociación particular, de los polimorfismos de 5-HTTLPR y CRF-BPs 11 con la restricción alimentaria crónica. Dado que los DC tendrían una sensibilidad al estrés asociada a su condición alimentaria, esta vulnerabilidad podría tener su origen en disposiciones genéticas específicas. El estudio presente tiene como objetivo evaluar preliminarmente esta hipótesis. Por ello, se explora si la presencia de los polimorfismos de 5-HTTLPR y CRF-BPs11 se asocia con la condición de DC.

\section{Material y Método}

\section{Participantes}

Fueron incluidos 132 individuos de sexo femenino, no relacionados entre sí, sin antecedentes de patologías psiquiátricas ni consumo de fármacos psicotrópicos, con edades comprendidas entre 18-25 años. Sólo se invitó a participar a mujeres al estudio dado que los modelos conceptuales y los estudios experimentales desarrollados en el ámbito de la restricción alimentaria crónica se han realizado sobre dicho grupo de personas ${ }^{2}$. 
Las participantes aceptaron mediante firma de un consentimiento informado escrito aprobado por el Comité de Ética Científica de la Facultad de Medicina, Universidad de La Frontera, Chile.

\section{Procedimiento}

Las participantes acudieron al laboratorio y respondieron un set de instrumentos psicométricos así como una prueba conductual asociados a un estudio amplio en desarrollo. Se les clasificó en dos grupos, dietante crónico o no dietante, aplicando la versión en español de la escala revisada de restricción alimentaria $(E R A)^{27-29}$. La ERA es un auto-reporte que evalúa actitudes hacia la alimentación, frecuencia de dietas y fluctuación de peso, permitiendo su clasificación de los participantes en dietantes crónicas y no dietantes (punto de corte de 12).

Además, se les extrajo una muestra de sangre venosa anticoagulada con EDTA mediante técnicas de enfermería habituales, siguiendo normas de bioseguridad según las recomendaciones descritas en el Manual de Normas de Bioseguridad de la Comisión Nacional Científica y Tecnológica (CONICYT).

\section{Análisis molecular}

Se extrajo ADN genómico de las participantes a partir de sangre total por el método de precipitación salina descrito por Salazar et $\mathrm{al}^{30}$. La genotipificación del polimorfismo 5-HTTLPR del gen SLC6A4 fue realizada mediante amplificación por la técnica de reacción en cadena de la polimerasa (PCR), utilizando partidores previamente descri$\operatorname{tos}^{16}$. Las condiciones de la PCR fueron: denaturación inicial a $98{ }^{\circ} \mathrm{C}$ por 5 minutos, seguido de 30 ciclos repetitivos compuestos por denaturación de 1 minuto a $95^{\circ} \mathrm{C}$, hibridación por 1 minuto a 61 ${ }^{\circ} \mathrm{C}$ y extensión de 1 minuto a $72{ }^{\circ} \mathrm{C}$, finalizando con una extensión de 10 minutos. Los fragmentos amplificados fueron visualizados mediante electroforesis en gel de agarosa al 3\% teñido con bromuro de etidio. El genotipo homocigoto $l / l$ fue identificado por la presencia de un único fragmento de $528 \mathrm{pb}$, para el genotipo homocigoto $s / s$ se observó un fragmento de $484 \mathrm{pb}$, mientras que para el genotipo heterocigoto $l / s$ se observaron ambos fragmentos (525pb y $484 \mathrm{pb}$ ).

Para la genotipificación de la variante CRFBPs11 del gen $C R F-B P$, se utilizó la técnica de PCR-RFLP utilizando condiciones previamente descritas ${ }^{25}$. Brevemente, el fragmento amplificado de $503 \mathrm{pb}$ fue sometido a restricción enzimática mediante la endonucleasa de restricción Taq I (Fermentas, Lituania), siguiendo el siguiente protocolo: $10 \mu \mathrm{L}$ de producto de PCR, $5 \mathrm{U}$ de la enzima de restricción, $2 \mu \mathrm{L}$ de tampón de reacción 10X; conteniendo este último: Tris- $\mathrm{HCl} 10 \mathrm{mM}$ ( $\mathrm{pH} 8,0$ a $37^{\circ} \mathrm{C}$ ), $\mathrm{MgCl}_{2} 5 \mathrm{mM}, \mathrm{NaCl} 100 \mathrm{mM}$ y BSA $0,1 \mathrm{mg} / \mathrm{mL}$ y agua destilada estéril suficiente para completar un volumen final de $20 \mu \mathrm{L}$. La mezcla fue incubada por 16 horas en baño termorregulado a $65^{\circ} \mathrm{C}$. Finalmente, los fragmentos de restricción fueron visualizados en gel de agarosa al 2\%. El genotipo homocigoto CC, fue identificado por la presencia de un único fragmento de $503 \mathrm{pb}$; el genotipo heterocigoto CT por fragmentos de 503, 325 y $178 \mathrm{pb}$, y el genotipo homocigoto TT por dos fragmentos de 325 y $178 \mathrm{pb}$.

La posibilidad de contaminación en los análisis moleculares fue excluida por la utilización de controles de reactivos en cada serie de amplificación. La correcta genotipificación de los polimorfismos fue confirmada mediante la repetición al azar del $20 \%$ de los análisis previamente realizados.

\section{Resultados}

\section{Escala revisada de restricción alimentaria}

Del total de 132 individuos, 41 participantes fueron clasificados como DC y las restantes 91 como no dietantes crónicos (NDC), ver Tabla 1 para estadísticos descriptivos.

\section{Análisis molecular}

\section{Polimorfismo 5-HTTLPR del gen SLC6A4}

La distribución de los genotipos del polimorfismo de 5-HTTLPR fue consistente con el equilibrio de Hardy-Weinberg en ambos grupos (DC: $\chi^{2}=0,52, p>0,05$; NDC: $\left.\chi^{2}=0,98, p>0,05\right)$. Al comparar la distribución de los genotipos entre dietantes crónicos y no dietantes se observan diferencias estadísticamente significativas $(\mathrm{p}=0,033)$ considerando un modelo de codominancia genética, siendo más frecuente la presencia del genotipo homocigoto ss en los individuos DC. Al analizar la frecuencia de los alelos $s$ y $l$, se observan, de igual forma, diferencias significativas entre ambos grupos $(\mathrm{p}=0,008)$, siendo más frecuente en los dietantes la presencia del alelo $s$ respecto al grupo 
Tabla 1. Estadísticos descriptivos. Diferencias en parámetros de interés entre dietantes crónicos (DC) y no dietantes (NDC)

\begin{tabular}{|lcccc|}
\hline & $\begin{array}{c}\text { NDC } \\
(\mathbf{n}=\mathbf{9 1})\end{array}$ & $\begin{array}{c}\text { DC } \\
(\mathbf{n}=\mathbf{4 1 )}\end{array}$ & $\begin{array}{c}\text { Total } \\
(\mathbf{n}=\mathbf{1 3 2})\end{array}$ & $\mathbf{p *}^{*}$ \\
\hline Edad (años) & $20,52 \pm 2,01$ & $21,17 \pm 2,73$ & $20,71 \pm 2,26$ & 0,17 \\
IMC $\left(\mathrm{kg} / \mathrm{m}^{2}\right)$ & $20,58 \pm 4,33$ & $22,28 \pm 7,16$ & $21,10 \pm 5,38$ & 0,17 \\
\hline ERA & $8,47 \pm 3,59$ & $19,12 \pm 3,93$ & $11,78 \pm 6,17$ & $<0,001$ \\
\hline
\end{tabular}

Nota: Media \pm SD. IMC = Índice de masa corporal; ERA=Escala de restricción alimentaria. * Test $t$ de Student.

Tabla 2. Distribución genotípica y frecuencia relativa de alelos del polimorfismo 5HTTLPR del gen SLC6A4 en individuos dietantes y no dietantes

\begin{tabular}{|c|c|c|c|c|c|c|c|c|c|}
\hline \multirow[b]{3}{*}{ No dietantes } & \multirow[b]{3}{*}{ (91) } & \multicolumn{6}{|c|}{ Genotipos, \% } & \multicolumn{2}{|c|}{ Alelos } \\
\hline & & \multicolumn{2}{|c|}{$\mathbf{s} / \mathbf{s}$} & \multicolumn{2}{|c|}{$\mathbf{s} / \mathbf{l}$} & \multicolumn{2}{|c|}{ I/I } & \multirow{2}{*}{$\begin{array}{c}\text { s } \\
0,54\end{array}$} & \multirow{2}{*}{$\begin{array}{c}\text { I } \\
0,46\end{array}$} \\
\hline & & 31,9 & (29) & 43,9 & (40) & 24,2 & (22) & & \\
\hline \multirow[t]{2}{*}{ Dietantes } & (41) & 53,7 & $(22)$ & 36,6 & (15) & 9,7 & (4) & 0,72 & 0,28 \\
\hline & & \multicolumn{6}{|c|}{$\chi^{2}=6,83 ; 2 \mathrm{gl} ; p=0,033$} & \multicolumn{2}{|c|}{$\chi^{2}=6,96 ; 1 \mathrm{gl} ; p=0,008$} \\
\hline
\end{tabular}

Variables analizadas con la prueba Chi-cuadrado $\left(\chi^{2}\right) ; g l=$ grados de libertad. Número de individuos se presenta en paréntesis.

Tabla 3. Distribución genotípica y frecuencia relativa de alelos del polimorfismo CRF-BPs11 del gen CRF-BP en individuos dietantes y no dietantes

\begin{tabular}{|c|c|c|c|c|c|c|c|c|c|}
\hline & & & & Genot & os, o & & & & \\
\hline & & & & & & & & $\mathbf{T}$ & C \\
\hline No dietantes & (91) & 5,5 & (5) & 37,4 & (34) & 57,1 & (52) & 0,24 & 0,76 \\
\hline Dietantes & (41) & 9,7 & (4) & 41,5 & (17) & 48,8 & (20) & 0,30 & 0,70 \\
\hline & & & & $238 ; 2$ & $p=$ & & & $\chi^{2}=1$, & $=0,280$ \\
\hline
\end{tabular}

Variables analizadas con la prueba Chi-cuadrado $\left(\chi^{2}\right) ; g l=$ grados de libertad. Número de individuos se presenta en paréntesis.

de no dietantes. La razón de disparidad (Odds Ratio, OR) asociada al alelo $s$ fue de 2,1 (IC 95\% $1,2-3,6)$, lo que sugiere que dicho alelo constituye un factor de riesgo para la condición de dietante crónico (Tabla 2).

Polimorfismo CRF-BPs11 del gen CRF-BP

La distribución de los genotipos para el polimorfismo CRF-BPs11 fue también consistente con el equilibrio de Hardy-Weinberg tanto en el grupo de no dietantes $\left(\chi^{2}=0,033, p>0,05\right)$, como en el grupo de dietantes crónicos $\left(\chi^{2}=0,019, \mathrm{p}>0,05\right)$. No se observaron diferencias estadísticamente significativas entre las distribuciones genotípicas $(\mathrm{p}=0,538)$. De igual forma, no hubo diferencias significativas en la frecuencia relativa de alelos entre los grupos estudiados ( $\mathrm{p}=0,280$, Tabla 3 ).

\section{Discusión}

El presente estudio exploró la asociación de los polimorfismos genéticos 5-HTTLPR y CRFBPs11 en individuos con la condición de DC. Los resultados mostraron una asociación solamente para el polimorfismo 5-HTTLPR. En éste último, al comparar el grupo de individuos clasificados como DC versus los NDC, se observó una mayor 
frecuencia del genotipo $s / s$ y una mayor presencia del alelo $s$ en DC. Así mismo, la presencia del alelo $s$ puede considerarse un factor de riesgo para esta condición $(\mathrm{OR}=2,1 ; \mathrm{IC}=1,2-3,6)$.

$\mathrm{Si}$ bien no existen reportes previos referentes a los DC, existen condiciones relacionadas y patologías de la conducta alimentaria en la que se presentan asociaciones con el polimorfismo de 5-HTTLPR. Por ejemplo, existen estudios recientes sobre el polimorfismo 5-HTTLPR, en los que se asocia la presencia del genotipo $s / s$ a las mujeres con alimentación emocional y sintomatología depresiva ${ }^{31}$. Un estudio en pacientes bulímicos y anoréxicos logró establecer que la presencia del genotipo $s / s$ y el alelo $s$ favorecen el desarrollo de estos trastornos alimentarios ${ }^{32}$. Así mismo, estudios de meta-análisis muestran que el alelo $s$ del polimorfismo 5-HTTLPR confiere riesgo para el desarrollo de trastornos de la alimentación, particularmente asociado a la anorexia nervio$\mathrm{sa}^{33,34}$. Finalmente, en un estudio que incluyó a 558 mujeres no se logró observar una asociación entre la presencia del genotipo $s / s$ y el trastorno por "atracón alimentario", aunque sí se demostró que la influencia del alelo $s$ lo hace más intenso ${ }^{35}$.

Considerando la hipótesis de sensibilidad al estrés ${ }^{5}$, es posible sostener preliminarmente a partir de estos datos y un reporte previo ${ }^{7}$ que los DC podrían ser sujetos con una configuración psicobiológica vulnerable, favoreciendo ante eventos estresantes respuestas fisiológicas más intensas y mayores dificultades en la modulación de su reactividad emocional. En este sentido, la sucesión de niveles de expresión de los problemas de alimentación (Figura 1) estarían asociados a una configuración psicobiológica de riesgo, que incluiría el polimorfismo 5-HTTLPR, conformando una diátesis para dichos trastornos.

Nuestro estudio no mostró asociación entre el genotipo homocigoto TT para el polimorfismo CRFBPs 11 y la condición de dietante crónico, ni tampoco para la presencia del alelo T. Sin embargo, las frecuencias genotípicas obtenidas en esta muestra de sujetos $(6,8 \%$ TT, $38,6 \%$ TC y $54,5 \%$ CC) difieren a los reportes previos, en los que se encontró asociación entre este polimorfismo y depresión mayor en población sueca (12\% CC, $47 \%$ TC y $41 \%$ TT) en una muestra de 135 sujetos (22), y en población belga (12\% TT, $42 \%$ TC y $46 \%$ TT), en una muestra de 182 sujetos $^{22}$. Cabe mencionar, que en estas poblaciones descritas el mestizaje es menor comparado con el que se presenta en nuestro país.

Es importante notar que la población chilena posee un carácter mestizo, influenciado fuertemente por la presencia de los pueblos originarios y por población de origen hispano. Se incluyen además poblaciones no despreciables con origen alemán, francés, italiano y suizo, entre otros. Trabajos previos realizados en población del sur de Chile $^{36,37}$, demuestran que aproximadamente el 90\% de los sujetos presenta algún haplogrupo característico de población amerindia (A, B, C o D). Sin embargo, en el presente estudio no se consideró esta caracterización de las participantes. Esta es una limitación relevante y es un aspecto sustancial a considerar en el diseño de futuros estudios.

El presente estudio puede considerarse un reporte seminal para la exploración del rol de diferentes polimorfismos asociados a la restricción alimentaria crónica, especialmente el polimorfismo 5-HTTLPR. En ese contexto, es importante que estudios futuros permitan diferenciar claramente la influencia de este polimorfismo en la respuesta emocional, la conducta alimentaria y la posible interacción mutua entre ambas influencias. El modelo de sensibilidad al estrés, el cual requiere mayor confirmación, puede encontrar en esta aproximación genético-conductual un sustento científico relevante para explicar la vulnerabilidad al estrés y la adversidad en pacientes con conductas alimentarias problemáticas.

Agradecimientos. Los autores agradecen la asistencia técnica de los Tecnólogos Médicos, $\operatorname{Dr}(\mathrm{c})$. Alejandro Cuevas y Dr(c). Nicolás Saavedra.

\section{Referencias}

1. Silva JR, Pizzagalli DA, Larson CL, Jackson DC, Davidson RJ. Frontal brain asymmetry in restrained eaters. J Abnorm Psychol 2002; 111: 676-81.

2. Silva JR. Sobrealimentación inducida por la ansiedad, Parte I: Evidencia conductual, afectiva, metabólica y endocrina. Ter Psicol 2007; 25: 112-34.

3. Silva JR. Restricción alimenticia y sobrealimentación: Un modelo de la neurociencia afectiva. Rev Med Chile 2008; 136: 1336-42.

4. Silva JR, Ortiz M, Quiñones A, Vera P, Slachevsky A. Affective style and eating disorders: A field study. Eat Weight Disord 2011; 16: 73-80.

5. Silva JR. Un modelo psicobiológico de la sobrealimen- 
tación inducida por estrés. Revista de Psicología. 2009; 13 (2): 43-63.

6. Silva JR. Sobrealimentación inducida por la ansiedad, Parte II: Un marco de referencia neurocientífico para el desarrollo de técnicas psicoterapéuticas. Ter Psicol 2008; 26: 99-115.

7. Silva JR. Restraint eating and sensitivity to stress: Preliminary experimental evidence. Riv Psichiatr 2011; 46: 1-5.

8. Canli T, Lesch KP. Long story short: the serotonin transporter in emotion regulation and social cognition. Nat Neurosci 2007; 10 (9): 1103-9.

9. Contesse V, Lefebvre H, Lenglet S, Kuhn JM, Delarue C, Vaudry H. Role of 5-HT in the regulation of the brainpituitary-adrenal axis: effects of 5-HT on adrenocortical cells. Can J Physiol Pharm 2000; 78 (12): 967-83.

10. Lowry CA. Functional subsets of serotonergic neurones: implications for control of the hypothalamic-pituitaryadrenal axis. J Neuroendocrinol 2002; 14 (11): 911-23.

11. Liposits Z, Phelix C, Paull WK. Synaptic interaction of serotonergic axons and corticotropin releasing factor (CRF) synthesizing neurons in the hypothalamic paraventricular nucleus of the rat. A light and electron microscopic immunocytochemical study. Histochemistry 1987; 86 (6): 541-9.

12. Heisler LK, Pronchuk N, Nonogaki K, Zhou L, Raber J, Tung L, et al. Serotonin activates the hypothalamicpituitary-adrenal axis via serotonin $2 \mathrm{C}$ receptor stimulation. J Neurosci 2007; 27 (26): 6956-64.

13. Lesch KP, Zeng Y, Reif A, Gutknecht L. Anxiety-related traits in mice with modified genes of the serotonergic pathway. Eur J Pharmacol 2003; 480 (1-3): 185-204.

14. Munafo MR, Freimer NB, Ng W, Ophoff R, Veijola J, Miettunen J, et al. 5-HTTLPR genotype and anxietyrelated personality traits: a meta-analysis and new data. Am J Med Genet B Neuropsychiatr Genet 2009; 150B (2): 271-81.

15. Munafo MR, Clark TG, Moore LR, Payne E, Walton R, Flint J. Genetic polymorphisms and personality in healthy adults: a systematic review and meta-analysis. Mol Psychiatry 2003; 8 (5): 471-84.

16. Lesch KP, Bengel D, Heils A, Sabol SZ, Greenberg BD, Petri S, et al. Association of anxiety-related traits with a polymorphism in the serotonin transporter gene regulatory region. Science 1996; 274 (5292): 1527-31.

17. Heils A, Teufel A, Petri S, Stober G, Riederer P, Bengel D, et al. Allelic variation of human serotonin transporter gene expression. J Neurochem 1996; 66 (6): 2621-4.

18. Little KY, McLaughlin DP, Zhang L, Livermore CS, Dalack GW, McFinton PR, et al. Cocaine, ethanol, and genotype effects on human midbrain serotonin trans- porter binding sites and mRNA levels. Am J Psychiatry. 1998; 155 (2): 207-13.

19. Hu XZ, Lipsky RH, Zhu G, Akhtar LA, Taubman J, Greenberg BD, et al. Serotonin transporter promoter gain-of-function genotypes are linked to obsessivecompulsive disorder. Am J Hum Genet 2006; 78 (5): 815-26.

20. Bale TL, Vale WW. CRF and CRF receptors: role in stress responsivity and other behaviors. Annu Rev Pharmacol Toxicol 2004; 44: 525-57.

21. Potter E, Behan DP, Fischer WH, Linton EA, Lowry PJ, Vale WW. Cloning and characterization of the cDNAs for human and rat corticotropin releasing factor-binding proteins. Nature 1991; 349 (6308): 423-6.

22. Van Den Eede F, Van Broeckhoven C, Claes SJ. Corticotropin-releasing factor-binding protein, stress and major depression. Ageing Res Rev 2005; 4 (2): 213-39.

23. Behan DP, Potter E, Lewis KA, Jenkins NA, Copeland $\mathrm{N}$, Lowry PJ, et al. Cloning and Structure of the Human Corticotropin Releasing Factor-Binding Protein Gene (Crhbp). Genomics 1993; 16 (1): 63-8.

24. Vamvakopoulos NC, Sioutopoulou TO, Durkin SA, Nierman WC, Wasmuth JJ, Mcpherson JD. Mapping the Human Corticotropin-Releasing Hormone-Binding Protein Gene (Crhbp) to the Long Arm of Chromosome-5 (5q11.2-Q13.3). Genomics 1995; 25 (1): 325-7.

25. Van Den Eede F, Venken T, Del-Favero J, Norrback KF, Souery D, Nilsson LG, et al. Single nucleotide polymorphism analysis of corticotropin-releasing factor-binding protein gene in recurrent major depressive disorder. Psychiat Res 2007; 153 (1): 17-25.

26. Karolyi IJ, Burrows HL, Ramesh TM, Nakajima M, Lesh JS, Seong E, et al. Altered anxiety and weight gain in corticotropin-releasing hormone-binding protein-deficient mice. P Natl Acad Sci USA 1999; 96 (20): 11595-600.

27. Silva JR. Consistencia interna y validez factorial de la versión en español de la escala revisada de restricción alimentaria. Rev Chil Nutr 2010; 37: 41-9.

28. Silva JR, Livacic-Rojas P, Slachevsky A. Diferencias individuales en dietantes crónicos; Influencia de los sistemas motivacionales en la alimentación. Rev Med Chile 2006; 134: 735-42.

29. Silva JR, Urzúa A. Propiedades psicométricas de la versión en español de la escala revisada de restricción alimentaria en una muestra de adolescentes. Univ Psychol 2010; 9: 535-44.

30. Salazar L, Melo C, Cavalli S, Hinuy H, Hirata M, Hirata R. Micrométodo para extração de DNA genômico útil no diagnóstico molecular da hipercolesterolemia familial. Revista Brasileira de Análisis Clínicas 2001; 33 (3): 111-6.

31. van Strien T, van der Zwaluw CS, Engels RC. Emotional 
eating in adolescents: a gene (SLC6A4/5-HTT) - depressive feelings interaction analysis. J Psychiatr Res 2010; 44 (15): 1035-42.

32. Di Bella DD, Catalano M, Cavallini MC, Riboldi C, Bellodi L. Serotonin transporter linked polymorphic region in anorexia nervosa and bulimia nervosa. Mol Psychiatry 2000; 5 (3): 233-4.

33. Calati R, De Ronchi D, Bellini M, Serretti A. The 5-HTTLPR polymorphism and eating disorders: A meta-analysis. Int J Eat Disord. 2010 Mar 5.

34. Gorwood P. Eating disorders, serotonin transporter polymorphisms and potential treatment response. Am J Pharmacogenomics 2004; 4 (1): 9-17.

35. Akkermann K, Nordquist N, Oreland L, Harro J. Sero- tonin transporter gene promoter polymorphism affects the severity of binge eating in general population. Prog Neuropsychopharmacol Biol Psychiatry 2010; 34 (1): 111-4.

36. Lagos J, Lemus J, Sierra F, Mella R, Fuentes F, Ocares $\mathrm{G}$, et al. Asociación de niveles de lípidos y haplogrupos Amerindios de DNA mitocondrial en individuos chilenos hipercolesterolémicos tratados con Atorvastatina. Rev Chil Cardiol 2010; 29 (2): 208-13.

37. Guzmán N, Lanas F, Salazar LA. Influence of Amerindian mitochondrial DNA haplogroups on thrombosis susceptibility and frequency of four genetic prothrombotic variants in Southern Chilean subjects. Clin Chim Acta 2010; 411 (5-6): 444-7. 